\title{
Analysis Gas Emission Vehicles in Makassar Region
}

\author{
Soetyono Iskandar ${ }^{1)}$ \\ MT, M.Pd., Lecturer Mechanical Engineering Universitas Negeri Makassar, Jl. Hertasning Utara No. IIIB No. \\ 15 Makassar, (Bapedalda Jakarta, 2000).
}

\begin{abstract}
Vehicles in Makassar region encompassed the most gas emission that must be at concentration rate, in this research it showed higher than limiting point from stated by the government. The objective of this research is to describe the condition of vehicles gas emission and the different gas concentration emitted by the vehicles, and the controlling efforts and strategies to overcome the emission. This research was done in six months in Makassar. This research included visibility survey method, with the population of this research was consisting of all the vehicles in Makassar city and sampling technique used incidental which took 146 vehicle units. The vehicles gas emission was measured in AVL Emission Tester type 4000, and the data were analyzed in descriptive and inferensial statistics. The result of this research showed that; (1) The gas emission were; $C O$, $\mathrm{CO}_{2}, \mathrm{HK}$ and $\mathrm{NO}_{2}$ of vehicles in Makassar city was higher than limiting point, except the CO, (2) The different concentration gas were; $\mathrm{CO}, \mathrm{CO}_{2}, \mathrm{HK}$ and $\mathrm{NO}_{2}$ emitted by the vehicles in Makassar city.
\end{abstract}

Key words: gas emission, vehicles, Makkasar City.

\section{Background}

Toward building for long distance Indonesia was build in sector industrial. At the region in Indonesia included Makassar. Building in sector industrial at Makassar region since 1993 where established PT Kawasan Industri Makassar (KIMA) at 1996. Kind of industrial development at Makassar region were industrial food, manufactore wood, rattan and leather.

The building sector industrial gave numerous changed at social, economy, culture and environmental. The changed can be seen increased the total manufacture, total vehicles, total population, etc. All of gave impact to the changed environmental condition. Environmental numerous impact was atmosphere.

Atmosphere quality, componen environmental was vey urgent at the resident. Because will be efforted to community health and pleasant. Increased population and decreased green belt as source $\mathrm{O}_{2}$ to influent decreased atmosphere quality at the region. "Emission factor at the region not only by total population, but by total industrial and vehicles" (Soemarwoto, 2004).

Geography Makassar region as a capital South Celebes was laid at the coordinat $119^{\circ} 24^{\prime} 17,38^{\prime \prime}$ BT and $5^{\circ} 8^{\prime} 6,19$ LS. Administrative, Makassar region area were $175,77 \mathrm{~km}^{2}$ or $0,28 \%$ included 14 district and consist of

143 village. Boundary Makassar region were as the next:

- North border with Maros district

- South border with Gowadistrict East border with Maros district

- West border with Makassar strait

Emission atmosphere problem similar internasional problem and have happened at progress Stated.

Like United Stated established policy beginning 1970-s for numerous emission atmosphere at the region. cases Los Angeles Smog since America constitution government brought out Clean Air Act 1971 and National Air Quality Standard (Moore, 2000). Reduced emission atmosphere at Indonesia effort with Program Blue Sky (Prolabi) by government Indonesia in the decree Minister of Environmental Stated No. 15/MENLH/4/1996 (Bapedal, 2001).

Atmosphere have to prevented fo community life and another human being as source natural. The surface the earth consist of the Gases at atmosphere were: nitrogen $\left(\mathrm{N}_{2}\right)$ and oxigen $\left(\mathrm{O}_{2}\right)$. Another gases were not much as, argon (Ar) carbon dioxida $\left(\mathrm{CO}_{2}\right)$, carbon monoxida (CO), hidrocarbon (HK), oxida nitrogen $\left(\mathrm{N}_{\mathrm{x}} \mathrm{O}_{\mathrm{y}}\right)$, and sulfur dioxida $\left(\mathrm{SO}_{2}\right)$. "The several gas above as, $\mathrm{CO}, \mathrm{CO}_{2}, \mathrm{Hk}$, and $\mathrm{SO}_{2}$ were poison gases"

(Darmono, 2001: 11). Poison gases mix in atmosphere as pollution source.

Pollution source at the nature and in constant source condition. Numerous pollution source influence human being were vehicles gas emission, because pollution source closely at the residence. Beside vehicles gas emission were uproar, vibration, dusty, and accident risk

Air pollutant as numerous atmosphere pollution source from vehicles. Very defective human being life. Many others component environmental suffered with its impact, impact accumulative arrange in PP Nomor 27 Tahun 1999 (Bapedal Regional III, 1999). 
Influent air pollution against healthty were irritation and dirty fellow through breathing and pneumonia because $\mathrm{N}_{\mathrm{x}} \mathrm{O}_{\mathrm{y}}, \mathrm{SO}_{2}$, ozon and others components. These gases pollution in a long time can be bronchitis, and pneumonia. Vehicles gas emission cause mutagenik, and canker (karsinogenik) (Sastrawijaya, 1991).

\section{Method Research}

This research with survey, investigated its data, and included quantitative research because investigation the data and its problem, so the research was decriptive comparative research.

\subsection{Design research}

Explanation :

Table 1 Design research

\begin{tabular}{|l|lll|}
\hline No. & \multicolumn{1}{l|}{$\mathrm{X}_{1}$} & $\mathrm{X}_{2}$ \\
\hline 1 & $\mathrm{X}_{1.1}$ & $\mathrm{X}_{2.1}$ & \\
2 & $\mathrm{X}_{1.2}$ & $\mathrm{X}_{2.2}$ & \\
3 & $\mathrm{X}_{1.3}$ & $\mathrm{X}_{2.3}$ & \\
- & - & - & \\
- & - & - & \\
$\mathrm{n}$ & $\mathrm{X}_{1 . \mathrm{n}}$ & $\mathrm{X}_{2 . \mathrm{n}}$ & \\
\hline
\end{tabular}

$\mathrm{X}_{1}=$ concentration emission gas vehicles were operation at Makassar region indicated on the street (Jl. Balaikota)

$\mathrm{X}_{2}=$ concentration emission gas vehicles were operation at Makassar region indicated on the street (Jl. Sultan Alaudin)

\subsection{Technics Analysis Data}

Data concentration emission gas vehicles indicated with Spektrofotometer to solve with analysis statistic descriptive, description data have collected asi: average, median, modus, standard deviation, minimal and maksimal with graphic histogram and distribution frequensi (Sudjana, 1996).

For testing hypothesis was used uji-t different two sample independent on two side. Before were done tested analysis and than tested hypothesis, because uji-t this to be statistic parametric. Tested analysis were test normality and test homogenity. Test normality used Chi-Kuadrat and test normality used test Bartlet (Sudjana, 1996).

\subsection{Condition Vehicles Gas Emission at Makassar Region}

\section{Result And Discuss}

Research data investigated by indicated vehicles gas emission at two place Balaikota and Sultan Alauddin Street in Makassar region was analysed and investigated.

Result analysis data vehicles gas emission were $\mathrm{CO}, \mathrm{CO}_{2}, \mathrm{HK}$ dan $\mathrm{NO}_{2}$ at $M a k a s s a r$ region can be seen as the next.

\section{Indicated result emission gas when has done First Balaikota Street}

a. Emission gas $\mathrm{CO}$

Concentration emission gas $\mathrm{CO}$ ard vehicles were variation from 0,00 till $10,39 \%$. Concentratiom average emission gas $\mathrm{CO}$ from 73 vehicles were $3,23 \%$, standard deviation were 3,17 , median 2,41 , and modus were 0,11 . Distribution its frequency can be at the Table 2 .

Table 2 Distribution frequency emission gas $\mathrm{CO}$

\begin{tabular}{|c|c|r|r|}
\hline No. & class Interval & \multicolumn{1}{|c|}{$\begin{array}{c}\text { Frequency } \\
\text { Absolut }\end{array}$} & \multicolumn{1}{c|}{$\begin{array}{c}\text { Frequency } \\
\text { Relative }\end{array}$} \\
\hline 1. & $0.00-1.48$ & 31 & 42.47 \\
\hline 2. & $1.49-2.97$ & 14 & 19.18 \\
\hline 3. & $2.98-4.46$ & 6 & 8.22 \\
\hline 4. & $4.47-5.95$ & 5 & 6.85 \\
\hline 5. & $5.96-7.44$ & 7 & 9.59 \\
\hline 6. & $7.45-8.93$ & 2 & 2.73 \\
\hline 7. & $8.94-0.42$ & 8 & 10.96 \\
\hline Total & & 73 & 10.0 .1 \\
\hline
\end{tabular}

From Table 2 analysis statistic result emission gas CO. 51 vehicles $(69,86 \%)$ was under the border group, 5 vehicles $(6,85 \%)$ was above the border group, 17 vehicles $(23,29 \%)$ was above the border group, so the conclution that emission gas $\mathrm{CO}$ indicated result at Balaikota Street variation beginning under the border group, 
at the border, and above the border group, numerous under the border group that government have confirm, was $4,5 \%$. Histogram frequency emission gas $\mathrm{CO}$ can be seen at figure 1 as the next.
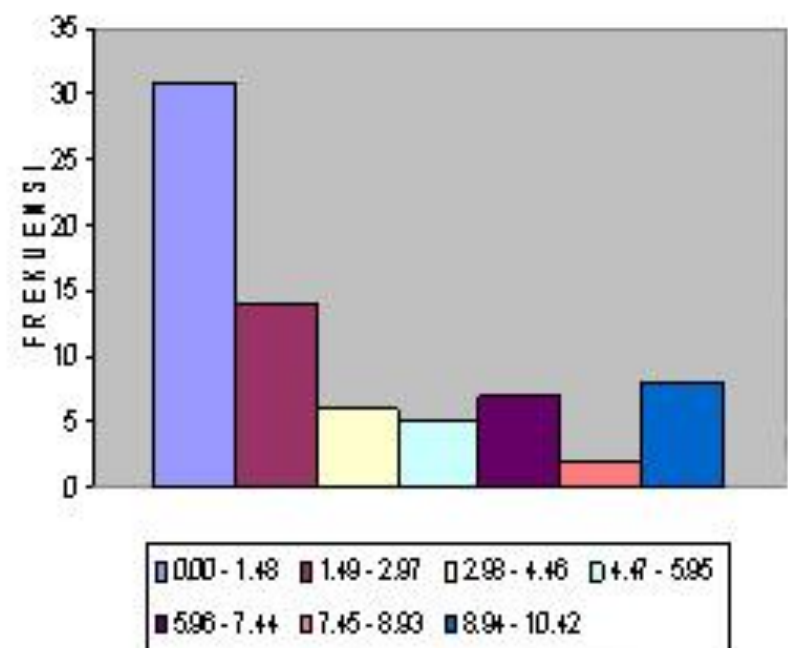

Figure 1 Histogram frequency emission gas $\mathrm{CO}$

\section{b. Emission gas $\mathrm{CO}_{2}$}

Concentration emission gas $\mathrm{CO}_{2}$ from vehicles were variation from 0,30 till 14,6\%. Concentration average emission gas $\mathrm{CO}_{2}$ from 73 vehicles were $10,65 \%$, standard deviation were 3,54, median 12,10, and modus were 13,60. Distribution frequency can be seen at the Table 3 .

Table 3 Distribution frequensi emission gas $\mathrm{CO}_{2}$

\begin{tabular}{|c|c|c|c|}
\hline No. & Class Interval & $\begin{array}{c}\text { Frequency } \\
\text { Absolut } \\
\end{array}$ & $\begin{array}{c}\text { Frequency } \\
\text { Relative }\end{array}$ \\
\hline 1. & $0.30-2.34$ & 4 & 5.48 \\
\hline 2. & $2.35-4.39$ & 2 & 2.74 \\
\hline 3. & $4.40-6.44$ & 2 & 2.74 \\
\hline 4. & $6.45-8.49$ & 11 & 15.07 \\
\hline 5. & $8.50-10.54$ & 11 & 15.07 \\
\hline 6. & -12.59 & 12 & 16.44 \\
\hline 7. & -14.64 & 31 & 42.46 \\
\hline \multicolumn{2}{|l|}{ Total } & 73 & 100.00 \\
\hline
\end{tabular}

Table 3 the result analysis statistic emission gas $\mathrm{CO}_{2}$, where 30 vehicles $(41,10 \%)$ were under the border group, 12 vehicles $(16,44 \%)$, were the border group 31 vehicles $(42,46 \%)$, were above the border group, so concluded that emission gas $\mathrm{CO}_{2}$ result indicated at Balaikota Street variation begin from under the border group, at the border group and at above the border group were government have confirm, were $12,00 \%$. Histogram frequency emission gas $\mathrm{CO}_{2}$ can be seen at the figure 2 as the next.

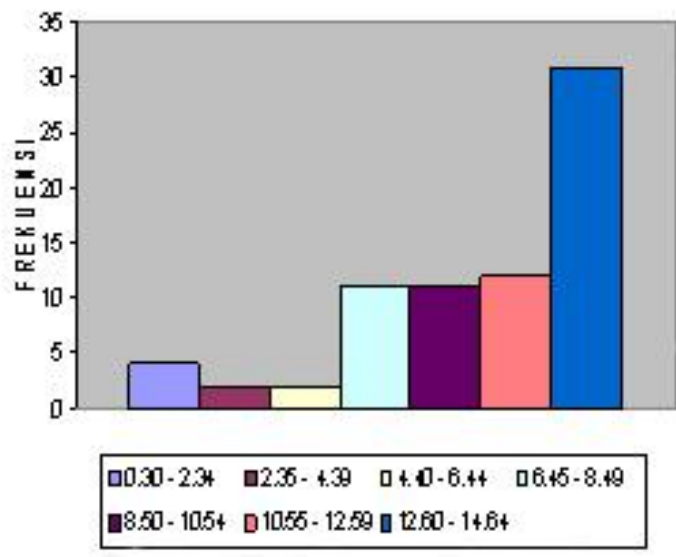

Figure 2 Histogram frequency emission gas $\mathrm{CO}_{2}$ 


\section{c. Emission gas HK}

Concentration emission gas HK from vehicles were variation from 19,00 till 3264,00 ppm. Concentration average gas HK from all vehicles were 689,04 ppm, standard deviation were 796,21, median 365,00 , modus were 107,00. Distribution frequency can be seen at Table 4 as the next.

Table 4 Distribution frequency emission gas HK

\begin{tabular}{|c|r|r|r|}
\hline No. & Class interval & $\begin{array}{c}\text { Frekuency } \\
\text { Absolut }\end{array}$ & $\begin{array}{c}\text { Frekuency } \\
\text { Relative }\end{array}$ \\
\hline 1. & $19.00-482.57$ & 43 & 58.90 \\
\hline 2. & $482.58-946.15$ & 17 & 23.29 \\
\hline 3. & $946.16-1409.73$ & 3 & 4.11 \\
\hline 4. & $1409.74-1873.31$ & 4 & 5.48 \\
\hline 5. & $1873.32-2336.89$ & 0 & 0 \\
\hline 6. & $2336.90-2800.47$ & 2 & 2.74 \\
\hline 7. & $2800.48-3264.05$ & 4 & 5.4 \\
\hline \multicolumn{2}{|l|}{} & 73 & 100.00 \\
\hline
\end{tabular}

Tabel 4 the result analysis statistic emission gas HK 60 vehicles $(82,19 \%)$ were under the border group, 3 vehicles $(4,11 \%)$ were the border, 11 vehicles $(13,70 \%)$ were above the border, so concluded that emission gas HK result indicated at Balaikota Street variation from were under the border, the border and above the border groups, numerous were above the border group that government have confirm, were $1000 \mathrm{ppm}$.

Histogram frequency emission gas HK can be seen at the figure 3 as the next.

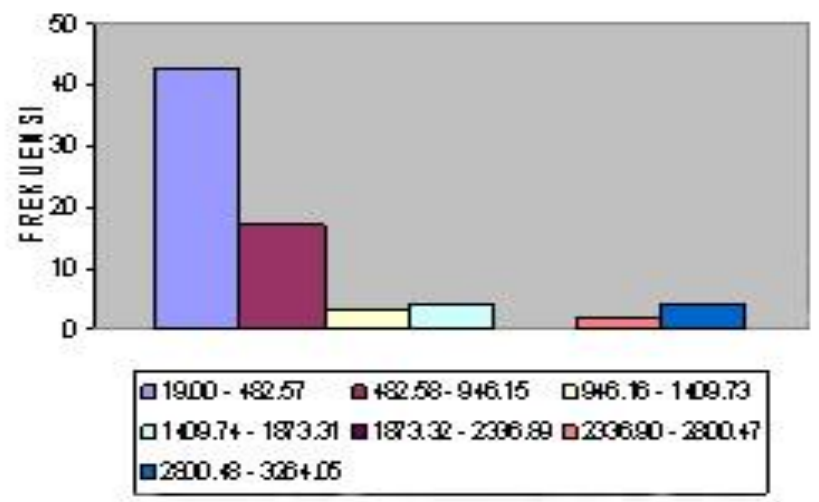

Figure 3 Histogram frequency emisi gas HK

\section{d. Emission gas $\mathrm{NO}_{2}$}

Concentration emission gas $\mathrm{NO}_{2}$ from vehicles variation from 33,00 till 100,00\%. Concentration average gas $\mathrm{NO}_{2}$ from all vehicles were $85,19 \%$, standard deviation were 17,51 , median 92,30, modus were 100,00. Distribution frequency can be seen at Table 5 as the next.

Table 5 Distribution frequency emission gas $\mathrm{NO}_{2}$

\begin{tabular}{|c|c|r|r|}
\hline No. & Class Interval & $\begin{array}{c}\text { Frequency } \\
\text { Absolut }\end{array}$ & $\begin{array}{c}\text { Frequency } \\
\text { Relative }\end{array}$ \\
\hline 1. & $33.30-42.57$ & 2 & 2,74 \\
\hline 2. & $42,58-52,15$ & 2 & 2,74 \\
\hline 3. & $52,16-61,73$ & 7 & 9,59 \\
\hline 4. & $61,74-71,31$ & 3 & 4,11 \\
\hline 5. & $71,32-80,89$ & 9 & 12,33 \\
\hline 6. & $80,90-90,57$ & 6 & 8,22 \\
\hline 7. & $90,58-100,05$ & 44 & 60,27 \\
\hline \multicolumn{2}{|c|}{ Total } & 73 & 100.00 \\
\hline
\end{tabular}

Table 5 the result analysis statistic emission gas $\mathrm{NO}_{2}, 2$ vehicles $(2,74 \%)$ were under the border group, 2 vehicles $(2,74 \%)$ were the border, 69 vehicles $(95,52 \%)$ were above the border group, so the concluded that emission gas $\mathrm{NO}_{2}$ variation from under the border, the border and above the border groups indicator quality maximal were government have confirm, were $50,00 \%$. 
Histogram frequency emission gas $\mathrm{NO}_{2}$ can be seen at the figure 4 as the next.

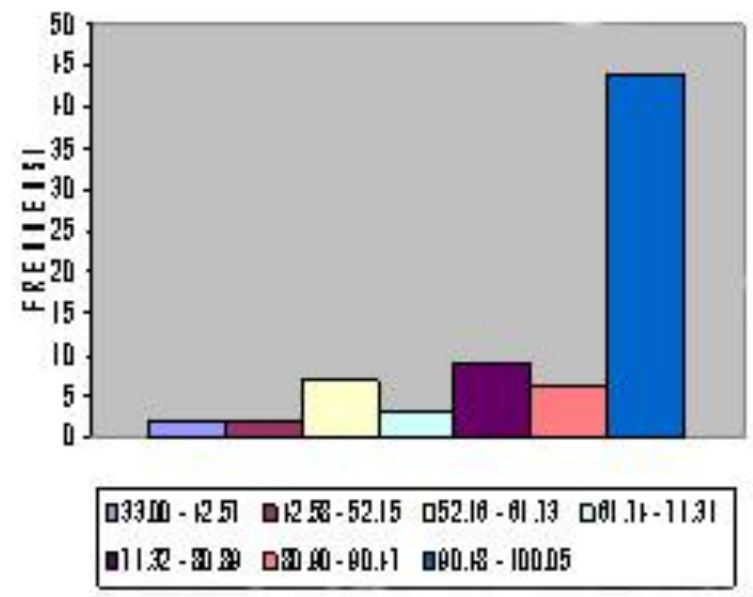

Figure 4 Histogram frequency emission gas $\mathrm{NO}_{2}$

\section{Result indicated emission gas investigated II at Sultan Alauddin Street}

a. Emission Gas CO

Concentration emission gas $\mathrm{CO}$ from vehicles were variation from 0,00 till $9,71 \%$. Concentration average gas CO from all the sample vehicles were $3,35 \%$, standard deviation were 3,10 , median 2,90, modus were 0,14 . Distribution frequention can be seen at the Table 6.

Table 6 Distribution frequency emission gas CO

\begin{tabular}{|c|r|r|r|}
\hline No. & Class Interval & $\begin{array}{c}\text { Frequency } \\
\text { Absolut }\end{array}$ & $\begin{array}{c}\text { Frequency } \\
\text { Relative }\end{array}$ \\
\hline 1. & $0.00-1.39$ & 28 & 38.36 \\
\hline 2. & $1.40-2.79$ & 8 & 10.96 \\
\hline 3. & $2.80-4.19$ & 12 & 16.44 \\
\hline 4. & $4.20-5.59$ & 5 & 6.85 \\
\hline 5. & $5.60-6.99$ & 8 & 10.96 \\
\hline 6. & $7.00-8.39$ & 4 & 5.47 \\
\hline 7. & $8.40-9.79$ & 8 & 10.96 \\
\hline Total & 73 & 100.00 \\
\hline
\end{tabular}

Table 6 the result analysis statistic emission gas $\mathrm{CO}$ were 48 vehicles $(65,75 \%)$ were under the border group, 5 vehicles $(6,85 \%)$ were the border group, 20 vehicles $(27,40 \%)$ were above the border group, so the concluded that emission gas CO result indicated at Sultan Alauddin Street variation from under the border, the border, and above the border groups, numerous were under the border that government have confirm, were $4,5 \%$.

Histogram frequency emission gas $\mathrm{CO}$ can be seen at the figure 5 as the next.

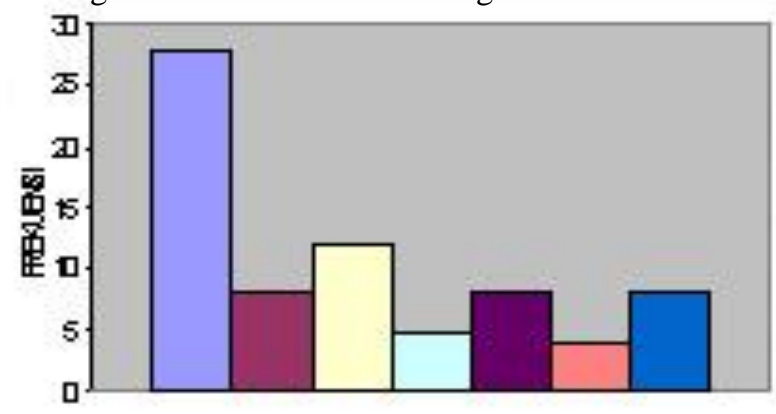

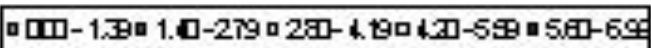

a 7 7 -870 8.0-9.9

Figure 5 Histogram frequency emission gas $\mathrm{CO}$ 


\section{b. Emission gas $\mathrm{CO}_{2}$}

Concentration emission gas $\mathrm{CO}_{2}$ From vehicles, variation from 0,60 till 13,80\%. Concentration average gas $\mathrm{CO}_{2}$ from all vehicles were $9,72 \%$, standard deviation were 3,11 , median 10,50 , modus were 9,10 . Distribution frequency can be seen at the Table 7 .

Table 7 Distribution frequency emission gas $\mathrm{CO}_{2}$

\begin{tabular}{|c|r|r|r|}
\hline No. & Class Interval & $\begin{array}{c}\text { Frequency } \\
\text { Absolut }\end{array}$ & $\begin{array}{c}\text { Frequency } \\
\text { Relative }\end{array}$ \\
\hline 1. & $0.60-2.49$ & 3 & 4.11 \\
\hline 2. & $2.50-4.39$ & 3 & 4.11 \\
\hline 3. & $4.40-6.29$ & 3 & 4.11 \\
\hline 4. & $6.30-8.19$ & 11 & 15.07 \\
\hline 5. & $8.20-10.09$ & 14 & 19.18 \\
\hline 6. & $10.10-11.99$ & 18 & 24.66 \\
\hline 7. & $12.00-13.89$ & 21 & 28.76 \\
\hline \multicolumn{2}{|l|}{} & 73 & 100.00 \\
\hline
\end{tabular}

Table 7 the result analysis statistic emission gas $\mathrm{CO}_{2}$ were 52 vehicles $(71,24 \%)$ were under the border group, 21 vehicles $(27,76 \%)$, were the border 0 vehicles $(0,00 \%)$ were above the border group, so the concluded that emission gas $\mathrm{CO}_{2}$ result indicated at Sultan Alauddin Street variation from were under the border, the border and above the border groups, numerous were under the border that the government have confirm, were $12,00 \%$.

Histogram frequency emission gas $\mathrm{CO}_{2}$ can be seen at the figure 6 as the next.

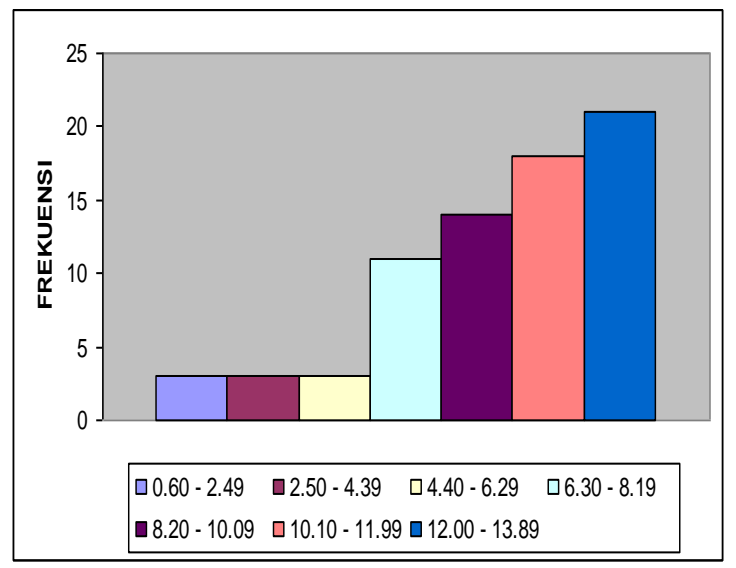

Gambar 6 Histogram frequency emission gas $\mathrm{CO}_{2}$

\section{c. Emission Gas HK}

Concentration emission gas HK from vechiles were variation from 50,00 till 4122,00 ppm. Concentration average gas HK from all vehicles were 951,62 ppm, standard deviation were 796,27, median 794,00 , modus were 1513,00. Distribution frequency can be seen at Table 8 .

Table 8 Distribution frequency emission gas HK

\begin{tabular}{|r|r|r|r|}
\hline No. & Class Interval & $\begin{array}{c}\text { Frekuency } \\
\text { Absolut }\end{array}$ & $\begin{array}{c}\text { Frequency } \\
\text { Relative }\end{array}$ \\
\hline 1. & $50.00-631,71$ & 32 & 43.84 \\
\hline 2. & $631.72-1213.43$ & 20 & 27.40 \\
\hline 3. & $1213.44-1795.15$ & 13 & 17.81 \\
\hline 4. & $1795.16-2376.87$ & 5 & 6.85 \\
\hline 5. & $2376.88-2958.59$ & 0 & 0.00 \\
\hline 6. & $2958.60-3540.31$ & 1 & 1.36 \\
\hline 7. & $3540.32-4122.03$ & 2 & 2.74 \\
\hline \multicolumn{2}{|l|}{ Total } & 73 & 100.00 \\
\hline
\end{tabular}


Table 8 the result analysis statistic emission gas HK 32 vehicles $(43,84 \%)$ were under the border group, 20 vehicles $(27,40 \%)$ were the border, 21 vehicles $(28,77 \%)$ were above the border, so the concluded that emission gas HK result indicated at Sultan Alauddin Street variation from under the border, the border and above the border, numerous were under the border that the government have confirm, were $1000 \mathrm{ppm}$.

Histogram frequency emission gas $\mathrm{HK}$ can be at the figure 7 as the next.

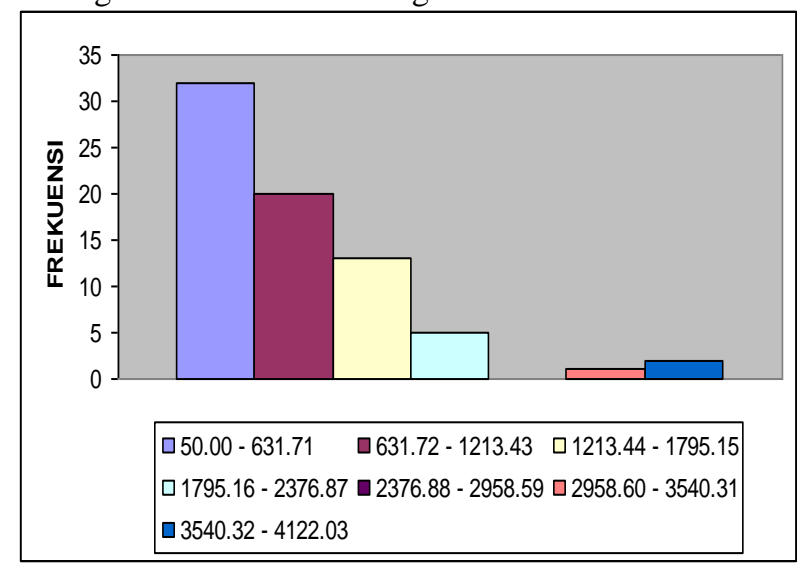

Figure 7 Histogram frequency emission gas HK

\section{d. Emission gas $\mathrm{NO}_{2}$}

Concentration emission gas $\mathrm{NO}_{2}$ from vehicles were variation from 16,40 till 100,00\%. Concentration average gas $\mathrm{NO}_{2}$ from all vehicles were $70,55 \%$, standard deviation were 24,69, median 76,60, modus were 75,30. Distribution frequency can be seen at the Table 9.

Table 9 Distribution frequency emission gas $\mathrm{NO}_{2}$

\begin{tabular}{|c|rr|r|r|}
\hline No. & Class Interval & $\begin{array}{c}\text { Frequency } \\
\text { Absolut }\end{array}$ & \multicolumn{1}{c|}{$\begin{array}{c}\text { Frequency } \\
\text { Relative }\end{array}$} \\
\hline 1. & 16.40 & $-28,34$ & 5 & 6.85 \\
\hline 2. & 28.35 & -40.29 & 9 & 12.33 \\
\hline 3. & 40.30 & -52.24 & 3 & 4.11 \\
\hline 4. & 52.25 & -64.19 & 9 & 12.33 \\
\hline 5. & 64.20 & -76.14 & 8 & 10.96 \\
\hline 6. & 76.15 & -88.09 & 17 & 23.29 \\
\hline 7. & 88.10 & -100.04 & 22 & 30.13 \\
\hline Total & & 73 & $17 \ldots \ldots \ldots \ldots \ldots \ldots$ \\
\hline
\end{tabular}

Table 9 the result analysis statistic emission gas $\mathrm{NO}_{2} 14$ vehicles $(19,14 \%)$ were under the border group, 3 vehicles $(4,11 \%)$ were the border group, 56 vehicles $(76,71 \%)$ were above the border group, so the concluded that emission gas $\mathrm{NO}_{2}$ result indicated at Sultan Alauddin Street variation from were under the border, the border and above the border groups indikator quality maximal were the government have confirm, were $50,00 \%$.

Histogram frequency emissin gas $\mathrm{NO}_{2}$ can be seen at the figure 8 as the next.

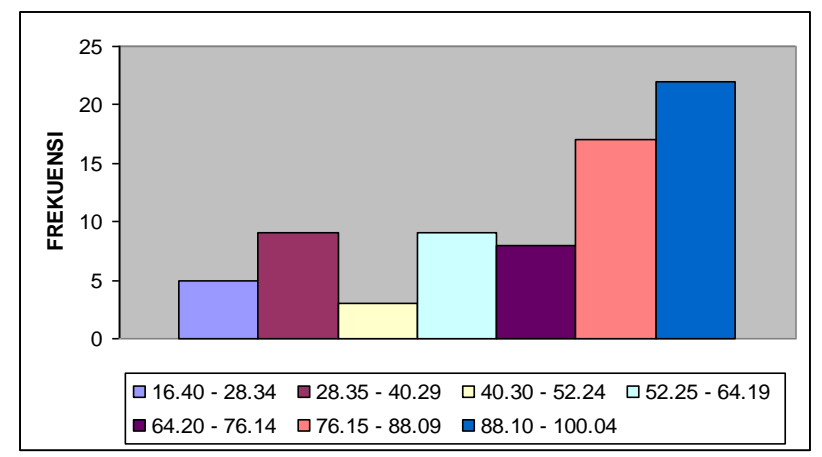

Figure 8 Histogram frequency emission gas $\mathrm{NO}_{2}$ 
Table 10Result test normality emission gases

\begin{tabular}{|c|c|r|r|r|l|}
\hline No & $\begin{array}{c}\text { Emissi } \\
\text { on } \\
\text { Gases }\end{array}$ & $X^{2}{ }_{\text {hit }(1)}$ & $X^{2}{ }_{\text {hit(2) }}$ & $X^{2}{ }_{(0,95 ; 6)}$ & Conclution \\
\hline 1. & $\mathrm{CO}$ & 55,01 & 38,34 & 12,6 & Berdis.Normal \\
\hline 2. & $\mathrm{CO}_{2}$ & 58,45 & 33,32 & 12,6 & Berdis.Normal \\
\hline 3. & $\mathrm{HK}$ & 136,29 & 82,6 & 12,6 & Berdis.Normal \\
\hline 4. & $\mathrm{NO}_{2}$ & 130,16 & 26,06 & 12,6 & Berdis.Normal \\
\hline
\end{tabular}

Table 11 Result test homogenity emission gases

\begin{tabular}{|c|c|c|c|c|}
\hline No. & $\begin{array}{c}\text { Emission } \\
\text { Gases }\end{array}$ & $X_{\text {hit }}^{2}$ & $X_{(0,95 ; 6)}^{2}$ & Conclution \\
\hline 1. & $\mathrm{CO}$ & 8,41 & 12,6 & Homogen \\
\hline 2. & $\mathrm{CO}_{2}$ & 8,46 & 12,6 & Homogen \\
\hline 3. & $\mathrm{HK}$ & 8,38 & 12,6 & Homogen \\
\hline 4. & $\mathrm{NO}_{2}$ & 9,17 & 12,6 & Homogen \\
\hline
\end{tabular}

Table 12 Result uji-t emission gases

\begin{tabular}{|c|l|r|r|l|}
\hline No. & \multicolumn{1}{|c|}{$\begin{array}{c}\text { Emission } \\
\text { Gases }\end{array}$} & \multicolumn{1}{|c|}{$\mathbf{t}_{\text {hit }}$} & $\mathbf{t}_{(\mathbf{0 , 9 5} ; \mathbf{7 1})}$ & \multicolumn{1}{c|}{ Result } \\
\hline 1. & $\mathrm{CO}$ & 0,2309 & 1,67 & The border \\
\hline 2. & $\mathrm{CO}_{2}$ & 1,70 & 1,67 & Above the border \\
\hline 3. & $\mathrm{HK}^{1}$ & 200,89 & 1,67 & Above the border \\
\hline 4. & $\mathrm{NO}_{2}$ & 4,13 & 1,67 & Above the border \\
\hline
\end{tabular}

\section{Conclution}

The result of these research with descriptive and inferensial, the conclution as the next :

1. Emission gas $\mathrm{CO}, \mathrm{CO}_{2}, \mathrm{HK}$ and $\mathrm{NO}_{2}$ vehicles at Makassar region variation begin from under the border, the border and above the border and numerous were above the border, excepted the emission gas $\mathrm{CO}$ numerous were under the border group that the government have confirm.

2. Found different concentration emission gas $\mathrm{CO}, \mathrm{CO}_{2}, \mathrm{HK}$ and $\mathrm{NO}_{2}$ were vehicles gases emission at Makassar region.

Conclution these research expression as suggestion to:

1. Government Makassar regional to the head of environmental and maker policyd to control the air pollution were that government have confirm.

2. The head of relationship as the routine evaluation vehicles gases emission, so that reduce the vehicles gas emission at Makassar region.

\section{Reference}

[1] Adji, C., 2003. "Bermimpi Indonesia Bebas Timbal”. Ozon. No. 4:24

[2] Abbas, Y., 2004. Analisis Emisi Gas Buang Kendaraan Bermotor Berbahan Bakar Bensin di Kota Makassar.

[3] Bapedal, 1997. Himpunan Peraturan di Bidang Pengendalian Dampak Lingkungan. Bapedal, Jakarta.

[4] Bapedalda Jakarta, 2000. Program Udara Bersih (Prodasih) DKI Jakarta Buku 1 NKLD Jakarta. http://bapedalda.dki.o.id/Buku1/main 5.htm).

[5] Diakses tanggal 13 Maret 2006.

[6] Bapedal, 2001. Himpunan Peraturan di Bidang Pengendalian Dampak Lingkungan. Bapedal, Jakarta.

[7] Bapedal Regional III, 1999. Peraturan Pemerintah Republik Indonesia Nomor 27 Tahun 1999 tentang Analisis Megenai Dampak Lingkungan Hidup. Bapedal Regional III, Makassar.

[8] Bapedal Regional III, 2001. Peraturan Pemerintah Republik Indonesia Nomor 41 Tahun 1999 tentang Pengendalian Pencemaran Udara. Bapedal Regional III, Makassar.

[9] Bapedalda Kota Makassar, 2001. Laporan Pelaksanaan Uji Petik Emisi Gas Buang Kendaraan Bermotor. Bapedalda Kota Makassar, Makassar.

[10] Darmono, C. 2001. Toksikologi Dasa, Asas, Organ Sasaran dan Penilaian Resiko. (Edisi kedua), UIP, Jakarta, hlm. 11.

[11] EPA2, 2000. Plobal. US-Environ Protection Agency (http://:www.epa.go/oar/globalwarming.nsf/content/Emissions.Html) Diakses tanggal 13 Maret 2006.

[12] Fardiaz, 1992. Polusi Air dan Udara. Kanisius, Jakarta.

[13] Kantor Menteri Negara LH, 1990. Kualitas Lingkungan hidup di Indonesia. PT Intermasa, Jakarta.

[14] Kementerian Lingkungan Hidup, 2002. Program Langit Biru dan Konservasi Energi. http://bsllak.freeyellow.com/ENERGY.html) Diakses tanggal 13 Maret 2006.

[15] Keputusan Menteri Negara LH No. 35 Tahun 1993. tentang Ambang Batas Emisi Gas Buang Kendaraan Bermotor

[16] Himpunan Peraturan di Bidang Pengendalian Dampak Lingkungan. Bapedal, Jakarta.

[17] Kompas, 8 Juli 2001. Timbal hanya berkurang, pencemaran jalan terus

[18] Kompas, 28 Agustus 2001. Kewajiban baru pemilik mobil penumpang pribadi di Provinsi DKI Jakarta.

[19] Kompas, 12 Maret 2002, Mesin besar tidak harus berarti boros bensin

[20] Leggett, J. 1990a. "The Nature of the Greenhouse Threat". Dalam J. Leggett (Ed), Global Warming the Greenpeace Report. Oxford University Press, hlm. 14-43

[21] Leggett, J. 1990b. “Global Warming: a Greenpeace View”. Dalam J. Leggett (Ed), Global Warming the Greenpeace Report. Oxford University Press, hlm. 457-480. 
[22] Moore, C.2000. Mutu Udara Kota. Makalah Hijau Kedutaan Besar Amerika (http://:www.usembassyjakarta.org/ptp/udarkt3.html) Diakses tanggal 13 Maret 2006.

[23] Ryadi, A.L.S., 1986. Pengantar Kesehatan Lingkungan. Karya Anda, Surabaya.

[24] Sarwono, S.W. 1992. Psikologi Lingkungan. Gramedia Widia Sarana Indonesia, Jakarta.

[25] Sastrawijaya, A.T. 1991. Pencemaran Lingkungan. Penerbit Rineka Cipta, Jakarta.

[26] Soemarwoto, Otto. 2004. Analisis Mengenai Dampak Lingkungan. Gadjah Mada University Press, Yogyakarta, hlm. 259.

[27] Sudjana, 1996. Metoda Statistika. Edisi ke-6. Tarsito, Bandung, hlm. 4494, 261-273.

[28] Sumriyatna, 2000. Bensin Tanpa Timbal.

[29] (http://www.anto2000.astra.co.id/berita/HotNewsDetail.asp?ID=16) Diakses 13 Maret 2006.

[30] Utaminingtyas, 2003. “Bensin Tanpa Timbal Merusak Mesin?”Ozon. No. 4, hlm. 19-20. 